\title{
STUDY OF THE EFFECT OF THE AQUEOUS SOLVATION ON THE GEOMETRIC PARAMETERS OF THE MELATONIN USING CAR-PARRINELLO MOLECULAR DYNAMICS
}

\author{
Allane C. C. Rodrigues ${ }^{1}$ (PG)*, Lilian T. F. de M. Camargo ${ }^{1,2}$ (PG), Dr. Ademir J. \\ Camargo $^{1}(\mathrm{PQ})$. \\ allane.c.c.rodrigues@gmail.com \\ 1 - Universidade Estadual de Goiás, CCET.BR 153 Quadra Área km99, Anápolis - GO. 75132-903. \\ 2 - Instituto Federal de Goiás, Av. Pedro Ludovico, S/N - Residencial Reny Cury, Anápolis - GO, 75131- \\ 457. \\ Keywords: Melatonin, Ab-Initio Molecular Dynamics, Car-Parrinello Molecular Dynamics.
}

\section{Introduction}

Melatonin (5-methoxy-N-acetyltryptamine) is a hormone produced by the pineal gland from amino acid tryptophan (1). Once released into the bloodstream, melatonin presents several different types of bioactivities. Among them, we can highlight the ability to remove free radicals, antioxidant activities, DNA protection, circadian cycle regulation, etc (2). Despite their physiological importance, the mechanism underlying its effects is not yet fully understood. It is possible that the broad spectrum of their bioactivities is related to the interaction of melatonin with the cell membranes (3). Therefore, the interaction of melatonin with water molecules present in the bloodstream is of fundamental importance to understand its bioactivity. However, studies of the interaction of melatonin with water molecules are quite limited in the literature. In this sense, the present work aims to study the effects of the aqueous solvation on the geometric and electronic parameters of melatonin using Car-Parrinello Molecular Dynamics.

\section{Methodology}

The Car-Parrinello Molecular Dynamics (CPMD) simulation was carried out using the CPMD program package (Version 4.1). In order to quantify the effects of aqueous solvation on the geometric and electronic parameters of melatonin, two systems were built: a system formed by a cubic box of $16 \AA$ with only one isolated melatonin molecule (System I) and another system formed by a cubic box of $16 \AA$ with one melatonin molecule and 100 water molecules (System II), simulating the condition of infinite dilution. The ionic temperature and the fictitious electronic kinetic energy were controlled using a chain of 3 Nose-Hoover thermostats. The ionic temperature of the two systems was set at $300 \mathrm{~K}$ in the canonical ensemble (NVT) with periodic conditions. The dynamic's step was fixed in 5 atomic units of time for both equilibration and production. The fictitious mass $(\mu)$ employed was 400 mass atomic units. The exchange-correlation functional PBE and Vanderbilt ultrasoft pseudopotentials were used to describe core-valence electron interactions. The wave functions of the valence electrons were expanded using plane waves with the cutoff energy of $25 \mathrm{Ry}$. The properties of interest were analyzed using the gqtea program developed by the theoretical and structural chemistry group of Anápolis.

\section{Results and Discussion}

The simulation time for System I was 50ps, while for System II the simulation time was 38ps. Figure 1 shows the structure of the melatonin molecule, as well as the respective numbering adopted in the calculations. The calculations of the absolute percentage differences for the geometric parameters were performed using the following Equation 


$$
D a=\frac{\left|\chi_{I}-\chi_{I I}\right|}{\chi_{I}} \times(100 \%)
$$

where $\chi_{I}$ represents the mean value obtained for the system I and $\chi_{I I}$ represents the mean value obtained for the system II.

Figure 1: Graphical representation of melatonin with the respective atomic numbering adopted in the calculations.

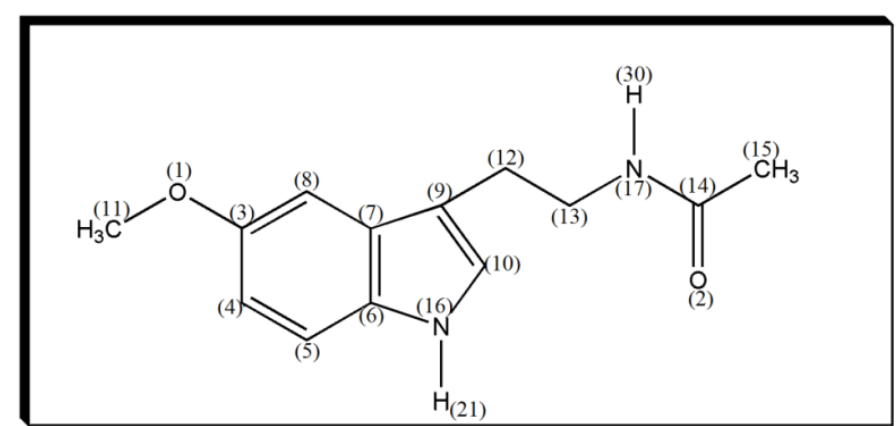

The main effects of aqueous solvation on geometric parameters of melatonin were observed for the bond lengths C14-O2 (2.176\%), N17-C14 (2.169\%) and N16$\mathrm{H} 21(1,079 \%)$. The changes for the bond lengths are mainly due to the strong hydrogen bonds observed at the $\mathrm{O} 2$ and $\mathrm{H} 21$ sites with water molecules, which change the electronic densities of these bonds. The bond angles that were most affected by aqueous solvation were O1-C3-C4 e O1-C3-C8 which an absolute average deviation of $4.37 \%$ and $4.241 \%$, respectively. These deviations of the aqueous phase relative to the gas phase are attributed to the hydrogen bonding at the $\mathrm{O} 1$ site of the methoxy group. Significant variations were also observed for the bond angles C7-C9-C12 (1.165\%), C9C12-C13 (2.590\%), C10-C9-C12 (1.320\%), H27-C12-C13 (1.328\%). Such changes are attributed to the effect of aqueous solvation of the amid group and indole $\mathrm{NH}$ group of the melatonin molecule with water.

\section{Conclusion}

The results of the Car-Parrinello Molecular Dynamics (CPMD) calculations show that the aqueous solvation significantly affects the geometric and electronic parameters of melatonin. These effects are attributed mainly to the hydrogen bonding formations occurring at $\mathrm{O} 1, \mathrm{O} 2, \mathrm{H} 30$, and $\mathrm{H} 21$ sites. Hence, these geometric and electronic changes should be taken into the account to explain the bioactivities of melatonin.

\section{Acknowledgment}

The authors are grateful for the financial support from the Coordenação de Aperfeiçoamento de Pessoal de Nível Superior (CAPES).

This research was developed with the support of the High-Performance Computing Center at the Universidade Estadual de Goiás.

\section{Reference}

1- Aaron B. Lerner, et, al. Structure of melatonin. Journal of the American Chemical Society. v. 81, p. 6084-6085, 1959.

2- SakirErkoç, et, al. Theoretical investigation of melatonin and its hydroxy isomers. Journal of Molecular Structure: THEOCHEM. v. 587, Issue. 1-3, p. 73-79, 2002.

3- FerideSevercan, et, al. Melatonin strongly interacts with zwitterionic model membranes-evidence from Fourier transform infrared spectroscopy and differential scanning calorimetry. Biochimica et Biophysica Acta. v. 1668, Issue. 2, p. 215-222, 2005. 